3

4

5

6

\title{
The role of task and ego-oriented climate in explaining students' bright and dark motivational experiences in Physical Education.
}

\section{Luis García-González ${ }^{a}$, Javier Sevil-Serranoa ${ }^{\text {, Angel Abós }}{ }^{a}$,}

Nathalie Aelterman ${ }^{b, c}$, and Leen Haerens ${ }^{b}$

aLuis García-González: Faculty of Health and Sport Sciences, University of Zaragoza, Spain. Email: Igarciag@unizar.es

a Javier Sevil-Serrano: Faculty of Health and Sport Sciences, University of Zaragoza, Spain. Email: jsevils@unizar.es

aÁngel Abós: Faculty of Health and Sport Sciences, Department of Didactics of the Musical, Plastic and Corporal Expression, University of Zaragoza, Spain. Email: aabosc@unizar.es

b,c Nathalie Aelterman: 'Department of Developmental, Personality and Social Psychology, Faculty of Psychology and EducationalSciences, Ghent University, Ghent (Belgium) bepartment of Movement and Sports Sciences, Faculty of Medicine and Health Sciences, Ghent University, Ghent (Belgium); Research Foundation Flanders (FWO), Belgium. Email: Nathalie.Aelterman@ugent.be

b Leen Haerens: Department of Movement and Sports Sciences, Faculty of Medicine and Health Sciences, Ghent University, Ghent (Belgium). Email: Leen.Haerens@ugent.be

Corresponding author: ${ }^{1}$ Luis García-González: Faculty of Health and Sport Sciences, University of Zaragoza, Spain. Plaza Universidad, 3, Huesca, 22001 (Spain). Email: lgarciag@unizar.es 
3 Background: A distinction is made in Achievement Goal Theory (AGT) between task-

4 oriented (i.e., effort, intra-individual progress, and self-comparison) and ego-oriented

5 (i.e., inter-individual progress and normative comparison) climates. Combining insights

6 from AGT and Self-Determination Theory (SDT), studies in the PE context have shown

7 that a task-oriented climate positively relates to need satisfaction, although the findings

8 regarding the motivating role of an ego-oriented climate are inconsistent. Moreover,

9 little is known about the role of task- or ego-oriented climates in explaining experiences

10 of basic psychological need frustration.

11 Purpose: Grounded in AGT and SDT, the aim of the present study was to examine if

12 experiences of basic psychological need satisfaction and need frustration can explain

13 why task- and ego-oriented climates elicit positive and negative motivational outcomes

14 in PE, respectively.

15 Research design: Cross-sectional study.

16 Method: A sample of 524 secondary school students $\left(51.1 \%\right.$ boys, $M_{\text {age }}=14.51 ; S D=$

17 1.81) from five different secondary schools participated in this study. Students reported

18 on their perceptions of task- and ego-oriented climates, motivational regulations, basic

19 psychological need satisfaction, and need frustration, as well as positive and negative

20 outcomes in PE. Structural equation modeling was used to investigate our objective.

21 Results: We found that a task-oriented climate had a strong and positive relationship

22 with basic psychological need satisfaction, eliciting a bright pathway to autonomous

23 motivation and affective attitude. An ego-oriented climate was positively related to

24 basic psychological need frustration, eliciting a dark pathway to amotivation and

25 boredom. A negative cross-path from task-oriented climate to basic psychological need 
1 frustration was also found, while no significant cross-paths were found from ego-

2 oriented climate to basic psychological need satisfaction.

3 Conclusions: This study provides a better understanding of the mechanisms that

4 explain why task- and ego-oriented climates shape students' motivational experiences in

5 PE lessons. It is suggested that a task-oriented climate elicits a bright pathway towards

6 more optimal functioning, because it fosters experiences of need satisfaction and buffers

7 against experiences of need frustration. In contrast, an ego-oriented climate is primarily

8 positively related to feelings of need frustration and negative motivational outcomes.

$9 \quad$ Practical implications for PE teacher training are discussed.

10

11 Keywords: Achievement goal; self-determination; motivational climate; motivation;

12 basic psychological need.

13

14

15

16

17

18

19

20 
2 pivotal role in determining students' motivational experiences (Braithwaite, Spray, and

3 Warburton 2011). When teaching and interacting with students, some teachers

4 concentrate more clearly on the students' personal learning progress. Others, however,

5 focus more explicitly on normative comparisons, thereby focusing on students' abilities

6 in relation to other students' performances (Ames, 1992). One theory that helps to

7 conceptualize and understand such differences between teaching strategies is

8 Achievement Goal Theory (AGT; Nicholls, 1989).

9 Motivational climate in PE.

10 According to the AGT, two types of motivational climate can be distinguished.

11 In a task-oriented climate, the teacher focuses on students' self-referenced or intra-

12 individual progress and effort. In contrast, in an ego-oriented climate, the teacher

13 focuses on social or normative comparison, inter-individual performance (i.e., social

14 comparison), and public recognition of success and failure (Nicholls, 1989). For

15 example, a teacher could assess the performance of his/her students in a 400-meter race

16 by assessing their improvement and comparing it to their own prior performances, (i.e.,

17 task-oriented climate) or by comparing the final time between students (i.e. ego-oriented 18 climate).

19 In the extant literature grounded in AGT (Nicholls, 1989), a task-oriented 20 climate has been related to adaptive outcomes such as more positive attitudes toward PE 21 (Erturan-İlker and Demirhan 2013), more enjoyment (Jaakkola et al. 2017), and greater 22 intention to be physically active (Di Battista et al. 2018). On the other hand, an ego23 oriented climate has been linked to negative and maladaptive outcomes such as 24 boredom and anxiety (Braithwaite et al. 2011).

25 Basic psychological need satisfaction and need frustration 
In the present study, the distinct underlying motivational processes explaining

2 the positive and negative effects of both motivational climates are further unraveled

3 from the perspective of Self-Determination Theory (SDT; Deci and Ryan 2000). Central

4 within SDT is the idea that students will thrive and learn most when teachers manage to

5 create a learning climate that supports their three basic psychological needs (BPN):

6 autonomy (e.g., students feel psychologically free and can actively participate in

7 decision-making), competence (e.g., students feel that they are effective) and

8 relatedness (e.g., students feel connected and experience a sense of belongingness with

9 their classmates or the teacher; Deci and Ryan 2000). In contrast, students will be

10 hampered in their learning when teachers create performance-oriented climates in which

11 students experience explicit feelings of need frustration (Haerens et al. 2015;

12 Vansteenkiste and Ryan 2013). Need frustration relates to feeling pressured or forced to

13 do things one would not choose to do (i.e., autonomy need frustration), feelings of

14 inferiority and failure (i.e., competence need frustration), and loneliness and alienation

15 (i.e., relatedness need frustration).

16 Recent evidence in PE indicates that teachers can catalyze a 'bright' pathway

17 toward more optimal functioning if they effectively nurture students' BPN for

18 relatedness, competence, and autonomy (e.g., Haerens et al. 2015; Sánchez-Oliva et al.

19 2014), while they can also elicit a 'dark’ pathway if their teaching strategies induce

20 feelings of need frustration (e.g., Haerens et al. 2015; Haerens et al. 2018; Liu,

21 Bartholomew, and Chung 2017). Moreover, need satisfaction and need frustration have

22 been found to be associated with different antecedents (e.g., need-supportive or need-

23 thwarting teaching behavior) in PE lessons (Haerens et al. 2015; Haerens et al. 2018),

24 suggesting that considering them as separate constructs is highly relevant. Related to

25 task- and ego-oriented climates, we therefore hypothesize in the current study that 
1 experiences of need satisfaction will explain why a task-oriented climate relates to more

2 adaptive outcomes, while experiences of need frustration will account for the

3 maladaptive effects of an ego-oriented climate.

4

We specifically suggest that a focus on intra-individual progress, which is characteristic of a task-oriented climate, provides opportunities to nurture the need for autonomy because a focus on detecting and honoring personal progress may lead students to more strongly endorse a personally meaningful learning trajectory. In addition, competence satisfaction is likely to be higher in a task-oriented climate, because a focus on progress better guarantees that all students, even those who are less capable than their peers, can improve their personal skills. Finally, if the main purpose is intrapersonal improvement, regardless of the ability of the class group, the need for relatedness is more likely to be satisfied. In contrast, relying on an inter-individual or performance-oriented approach (i.e., ego-oriented climate) may provide experiences of BPN frustration because students may be more likely to feel pressured to satisfy the teachers’ expectations to perform well (i.e., autonomy frustration), because only a few students can be the "best” performers (i.e., competence frustration), and because social comparison can elicit negative interpersonal dynamics among students (i.e., relatedness frustration) (Holt et al. 2018). In support of our premises, previous studies have shown that a task-oriented climate positively relates to autonomy, competence, and relatedness need satisfaction in PE (Di Battista et al. 2018) and in the sport context (Álvarez et al. 2012). Associations between an ego-oriented climate and need frustration, however, have largely remained understudied so far.

Furthermore, the few studies focusing on the relationship between an egooriented climate and the separate needs for autonomy, competence, and relatedness have reported inconsistent and mixed results. Specifically, in the study of Ommundsen and 
1 Kvalø (2007), an ego-oriented climate did not relate to perceived autonomy and

2 competence need satisfaction, yet relatedness satisfaction was not measured. Recently,

3 Cera et al. (2015) did not find any relationship between ego-oriented climate and

4 autonomy, competence, and relatedness need satisfaction, either. A previous study

5 conducted by Álvarez et al. (2012) in a sports setting showed that ego-oriented climate

6 was negatively related to perceived relatedness satisfaction, but it was not related to

7 perceived autonomy or competence satisfaction in a sample of young athletes.

8 Students' motivation and outcomes in physical education

According to the SDT, a better understanding of the antecedents of need

10 satisfaction is important, because the satisfaction of BPN fosters more self-determined

11 forms of motivation, whereas feelings of need frustration are suggested to be associated

12 with less self-determined forms of motivation in the PE domain (Sun, Li, and Shen

13 2017). Indeed, SDT distinguishes different forms of motivation on a continuum from

14 more to less self-determined forms of motivation (Deci and Ryan 2000). Intrinsic

15 motivation (e.g., students participate in an activity for the feeling of enjoyment and

16 pleasure) and identified regulation (e.g., students participate in an activity because it is

17 important or useful to them) represent the most self-determined or autonomous forms of

18 motivation (Deci and Ryan 2000). SDT also differentiates between introjected

19 regulation (e.g., students participate in an activity in order to avoid feelings of guilt and

20 shame) and external regulation (e.g., students participate in an activity to obtain rewards

21 or to avoid punishment) as two less self-determined or controlled forms of motivation.

22 Finally, amotivation refers to a lack of motivation or intentionality to take up an activity

23 (Deci and Ryan 2000).

24 In the specific context of PE, studies have demonstrated that autonomous

25 motivation is related to positive outcomes, such as enjoyment (Jaakkola et at. 2017), 
1 positive attitudes toward PE (Säfvenbom, Haugen, and Bulie 2015), future intentions to

2 exercise outside PE (Chen et al. 2014), and physical activity (PA) levels inside

3 (Aelterman et al. 2012) and outside PE (Haerens et al., 2010). Less self-determined

4 forms of motivation, on the other hand, were found to be negatively related to adaptive

5 outcomes such as rated class engagement (Aelterman et al. 2012), and positively related

6 to maladaptive outcomes such as boredom (Sánchez-Oliva et al. 2014). Further,

7 amotivation was found to be positively associated with maladaptive outcomes such as

8 boredom (Sánchez-Oliva et al. 2014) and unhappiness (Standage, Duda, and Ntoumanis

9 2005), while being negatively related to adaptive outcomes such as rated engagement

10 (Aelterman et al. 2012). Together, these studies show that students thrive when they are

11 autonomously motivated, while the opposite is true when they are motivated or

12 amotivated in a controlled manner.

\section{The present study}

14 Up until today, no studies in the context of PE have investigated the differential

15 role of task- and ego-oriented climates in relation to need frustration and students'

16 motivational experiences or affective outcomes in PE. The primary aim of the present

17 study was, therefore, to investigate whether processes of need satisfaction and

18 frustration can explain why task- and ego-oriented climates elicit distinct motivational

19 processes. As depicted in Figures 1 and 2, it was hypothesized that students' perceived

20 task-oriented climate would primarily relate to more self-determined forms of

21 motivation and positive affective outcomes via experiences of need satisfaction

22 (Hypothesis 1a), while perceived ego-oriented climate would primarily relate to less

23 self-determined motivation and amotivation, and negative affective outcomes via need

24 frustration (Hypothesis 1b). We also considered specific cross-path relationships (i.e.,

25 the relationship between one variable belonging to the bright pathway and another 
1 variable belonging to the dark pathway). It was specifically hypothesized that a

2 perceived task-oriented climate would be a buffer against need frustration (Hypothesis

3 2a), and also that a perceived ego-oriented climate would prevent experiences of need

4 satisfaction (Hypothesis 2b) (Vansteenkiste and Ryan 2013) (See Figures 1 and 2).

5 Consistent with previous studies in the PE context (Haerens et al. 2015; Liu et al. 2017),

6 we expected the theoretical symmetrical paths (e.g., between task-oriented climate and

7 need satisfaction), to be more strongly significant than the hypothetical cross-paths

8 (e.g., between task-oriented climate and need frustration) (Hypothesis 2c).

9 Finally, because an ego-oriented climate was found to be unrelated to autonomy 10 and competence satisfaction across studies (e.g., Álvarez et al. 2012; Ommundsen and 11 Kvalø 2007; Reinboth and Duda 2006; Smith et al. 2015), while one study found a 12 negative relationship to relatedness satisfaction (e.g., Alvarez et al. 2012), we also 13 wanted to investigate relationships between an ego-oriented climate and each of the 14 separate psychological needs for autonomy, competence, and relatedness (See Figure 2). [INSERT FIGURE 1, HERE] [INSERT FIGURE 2, HERE]

\section{Method}

18

Participants and procedure

Participants were 524 students (51.1\% were males) from 22 classes taught by 9

21 different teachers (each teacher taught between 1 and 5 classes) across 5 different 22 secondary schools in Spain. All participating teachers were full-time certified PE 23 teachers with a Master's degree in PE pedagogy. Students of these teachers filled out a 24 set of questionnaires at the end of a teaching unit. Questions concerned students' 25 perceptions of the motivational climate and their motivational experiences during the 26 teaching unit (e.g., rope skipping, acrosport, soccer, athletics, badminton) they had just 
1 participated in. The topic of the teaching unit was determined by the PE teacher, and the

2 duration of the teaching units was between 8 to 10 sessions. Students' ages ranged

3 between 12 and 19 years $(M=14.51 ; S D=1.81)$. Class sizes ranged between 21 and 30

4 students per class. A specific active consent to participate in the study was obtained

5 from each student's parents. An introductory letter explained the nature and objectives

6 of the study with a final agreement that was signed by the parents. Participation was

7 voluntary and student responses were anonymous and confidential. The University

8 Ethics Committee approved the study protocol.

9

Measures

All questionnaires were administered in paper-pencil format on a 5-point Likert

scale ranging between 1 (strongly disagree) to 5 (strongly agree).

Motivational climate.

Students completed a Spanish version (Gutiérrez, Ruiz, and López 2011) of the Perception of Motivational Climate Scale (PMCS; Biddle et al. 1995) to assess their perceptions of task- and ego-oriented climates in PE. For purposes of the present study, this scale was slightly adjusted to the specific teaching unit taught in each class (e.g.,

18 “In the acrosport teaching unit, our PE teacher...”). This statement was followed by 19 items divided into two factors: 9 items for task climate (e.g., "The PE teacher is pleased when everyone makes progress by putting effort into tasks”) and 10 items for ego-

21 oriented climate (e.g., “The PE teacher only bothers with those who do well in sport”).

22 Confirmatory Factor Analysis (CFA) showed good fit of the data to the two-factor 23 structure $\left(\chi^{2}(8)=20.273, p<.01 ; \chi^{2} / \mathrm{df}=2.53\right.$; RMSEA $=.05 ;$ SRMR $=.03$; CFI = .99;

24 TLI $=.98)$. The reliability analysis reflected Cronbach's alpha values of .90 for task25 oriented climate and .88 for ego-oriented climate.

26 Basic psychological need satisfaction. 
2 Exercise Scale (BPNES; Vlachopoulos \& Michailidou, 2006) was used to assess

3 students' experienced need satisfaction in PE. This instrument was adjusted to the

4 context of the study by modifying the stem and items to each teaching unit (e.g., "In the

5 skipping rope unit...”). This questionnaire comprises 12 items, grouped into 3 factors (4

6 items per factor) to measure: autonomy satisfaction (e.g., "I feel that I have the

7 opportunity to make choices with respect to the way I practice rope skipping”),

8 competence satisfaction (e.g., "I feel that rope skipping is an activity that I can do

9 well”), and relatedness satisfaction (e.g., "I feel that my peers interact with me in a

10 friendly way when I carry out the rope skipping unit”). CFA showed good fit of the data

11 to the three-factor structure $\left(\chi^{2}(51)=152.88, p<.001 ; \chi^{2} / \mathrm{df}=2.99\right.$; RMSEA $=.06$;

12 SRMR $=.05 ;$ CFI $=.94 ;$ TLI $=.92)$. The values of Cronbach's alpha coefficient were

$13.75, .77$ and .81 for autonomy, competence, and relatedness need satisfaction,

14 respectively.

15 Basic psychological need frustration.

16 Students' need frustration was measured by means of the Psychological Need

17 Thwarting Scale (PNTS; Bartholomew et al. 2011), which was translated into Spanish

18 (Sicilia, Ferriz, \& Sáenz-Álvarez 2012) and adjusted to the PE context. The stem of the

19 scale was adjusted to each teaching unit (e.g., "In the soccer unit...”). The instrument

20 consisted of 12 items distributed across 3 factors: (4 items per factor): autonomy

21 frustration (e.g., "I feel pushed to behave in certain ways”), competence frustration

22 (e.g., "Situations occur in which I feel incapable”), and relatedness frustration (e.g., "I

23 feel other people dislike me”). CFA showed good fit of the data to the three-factor

24 structure $\left(\chi^{2}(51)=110.78, p<.001 ; \chi^{2} / \mathrm{df}=2.17\right.$; RMSEA $=.05 ;$ SRMR $=.04 ;$ CFI $=$

25 .96; TLI $=.95)$. Internal reliability analysis showed acceptable alpha Cronbach values of 
1.76 , .82, and .80 for autonomy, competence, and relatedness need frustration,

2 respectively.

3 Motivational regulations.

$4 \quad$ The different motivational regulations were assessed using the Spanish version

5 (Martín-Albo, Nuñez, \& Navarro 2009) of the Situational Motivation Scale (SIMS;

6 Guay, Vallerand, \& Blanchard 2000). The stem "Why did you engage in this activity?"

7 was slightly adjusted to "Why did you engage in the teaching unit of...?”. The scale has

814 items distributed into 4 factors: intrinsic motivation (4 items, e.g., "Because this

9 activity is fun”), identified regulation (3 items, e.g., "Because I believe that this activity

10 is important for me”), external regulation (3 items, e.g., "Because I am supposed to do

11 it”) and amotivation (4 items, e.g., " I do this activity, but I am not sure it is a good thing

12 to pursue it”). Based on previous research (e.g., Haerens et al. 2015) autonomous

13 motivation was computed by obtaining the average from the subscales of intrinsic

14 motivation and identified regulation. Because this questionnaire did not include

15 introjected regulation it was not possible to calculate the broader dimension of

16 controlled motivation (sum of introjected regulation and external regulation) in the

17 current study. CFA showed good fit of the data to the four-factor structure $\left(\chi^{2}(71)=\right.$

18 199.73, $\left.p<.001 ; \chi^{2} / \mathrm{df}=2.81 ; \mathrm{RMSEA}=.06 ; \mathrm{SRMR}=.06 ; \mathrm{CFI}=.96 ; \mathrm{TLI}=.95\right)$. For

19 the current study, Cronbach’s alpha values were .91 for intrinsic motivation, .84 for

20 identified regulation, .79 for external regulation, and .87 for amotivation.

21 Affective outcomes.

22 In this study, boredom and affective attitude were measured as negative and 23 positive affective outcomes, respectively. The affective attitude subscale of the Physical

24 Education Predisposition Scale (PEPS; Hilland et al. 2009) was used to measure 25 affective attitude. This instrument contains 4 items (e.g., "The things I learn make 
1 lessons interesting for me”) and was translated into Spanish following the guidelines of

2 the International Test Commission (Muñiz, Elosua, and Hambleton 2013). Boredom

3 was measured by a subscale of the Sport Satisfaction Instrument (SSI; Duda and

4 Nicholls 1992) validated in Spanish in the context of PE (Baena-Extremera et al. 2012).

5 Boredom was comprised of 3 items (e.g., "In Physical Education classes, I am usually

6 bored”). The statement of both instruments was adapted to each teaching unit. CFA

7 showed good fit of the data to the two-factor structure $\left(\chi^{2}(13)=24.95, p<.001 ; \chi^{2} / \mathrm{df}=\right.$

8 1.92; RMSEA $=.04 ;$ SRMR $=.02 ; \mathrm{CFI}=.99 ; \mathrm{TLI}=.98)$. Cronbach's alpha values were

$9 \quad .81$ for boredom and .92 for affective attitude.

10 Statistical analysis

11 Means, standard deviations, and latent correlations were calculated as a first

12 step. CFA using maximum likelihood estimation and internal consistency analysis were

13 performed in order to test validity and reliability of the measures. Structural Equation

14 Modelling (SEM) was used to test the hypothesized models (see Figures 1 and 2). The

15 first model tested two symmetrical paths from task- and ego-oriented climates, via need

16 satisfaction and need frustration, to motivational experiences and affective outcomes. It

17 also tested specific hypothetical cross-paths (e.g., between task-oriented climate and

18 need frustration or between ego-oriented climate and need satisfaction; for further

19 detail, see Hypothesis 2a and 2b). In a second follow-up model, we tested two paths

20 from task- and ego-oriented climates to each of the BPN, separately, to obtain a more

21 fine-grained insight into different manifestations of motivational climate. Comparative

22 Fit Index (CFI), Tucker Lewis Index (TLI), and the Root Mean Squared Error of

23 Approximation (RMSEA) were used to evaluate the model fit. Following Hu and

24 Bentler (1999) recommendations, cut-off values close to .95 for CFI and TLI, and close

25 to .06 for RMSEA indicated good model fit. Latent constructs for task- and ego-oriented 
1 climates, need satisfaction and need frustration, as well as for autonomous motivation

2 were estimated based on three parcels calculated by the mean of each one. Latent

3 constructs for external regulation, amotivation, affective attitude, and boredom were

4 comprised of the items representing each scale. Data analyses were conducted by Mplus

$5 \quad 7$ and SPSS 20.0.

6 Results

7

8

9

In a first step, we investigated the role of task and ego-oriented climate in relation to need satisfaction and need frustration, and how these climates can elicit different motivational processes following a bright and dark pathway. Descriptive statistics and latent correlations between the variables of the first model are presented in Table 1. Correlations were in the expected direction with a task-oriented climate being significantly and positively related to BPN satisfaction, autonomous motivation, and affective attitude, while negatively related to BPN need frustration, amotivation, and boredom. An ego-oriented climate was significantly and negatively related to autonomous motivation and affective attitude, while being positively related to BPN frustration, external regulation, amotivation, and boredom.

As observed in Figure 3, results of the SEM analysis revealed that a taskoriented climate was strongly and positively related to BPN satisfaction, eliciting a bright pathway from BPN satisfaction to autonomous motivation. This bright pathway also continues with a positive relationship from autonomous motivation to affective attitude, and with a negative relationship from autonomous motivation to boredom. We also found evidence for a dark pathway, with an ego-oriented climate that was positively related to BPN frustration, which in turn related positively to amotivation. This dark pathway continues with a positive relationship between amotivation and boredom, and a negative relationship between amotivation and affective attitude. In 
1 addition, although a task-perceived oriented climate was primarily related to need

2 satisfaction, results showed a negative cross-path from a task-oriented climate to BPN

3 frustration. In contrast, no relationship was found between an ego-oriented climate and

4 BPN satisfaction. The tested model fit the data sufficiently $(\chi 2 / \mathrm{gl} .=3.35, p<.001$;

$5 \quad$ RMSEA $=.07 ;$ 90\% CI = .06-.07; CFI = .93; TLI = .92) (Marôco 2014).

6

7

8

9

10

\title{
[INSERT TABLE 1, HERE]
}

\section{[INSERT FIGURE 3, HERE]}

In a second step, we aimed to gain a deeper insight into the relationships between motivational climates and the three separate needs (i.e., autonomy, competence, and relatedness). Descriptive statistics and latent correlations among the variables of the second model are presented in Table 2. The results of these supplementary analyses, which are displayed in Figure 4, showed that a task-oriented climate was strongly and positively related to the satisfaction of all three BPN, and moderately and negatively related to the frustration of all three BPN. A perceived egooriented climate was positively related to the frustration of all three BPN and was negatively related to relatedness satisfaction. The results of the structural model indicated a good data fit $(\chi 2 / \mathrm{gl} .=3.09, p<.001$; RMSEA $=.06 ; 90 \% \mathrm{CI}=.06-.07 ;$ CFI $=.93 ;$ TLI $=.92)($ Marôco 2014).

\author{
[INSERT TABLE 2, HERE] \\ [INSERT FIGURE 4, HERE]
}

\section{Discussion}

This study aimed to understand why task- and ego-oriented climates exert a different motivational impact on the PE context by investigating their relationships with students' experiences of need satisfaction and need frustration. 
The "bright" side of motivation.

A task-oriented climate is characterized by teachers using statements such as

"You have to try to improve our own performance", "I am going to evaluate your progress”. In task-oriented climates teachers will more likely rely on private feedback and an evaluation focused on progress and self-referenced standards (e.g., "Sarah, well done, you managed to jump higher than last week. You are really improving your skills”). We hypothesized that students' perceived task-oriented climate would be primarily related to autonomous motivation and affective attitude via experiences of need satisfaction (Hypothesis 1a). Consistent with previous studies in PE lessons (Di Battista et al., 2018) and with our expectations, the present findings showed that students' perceived task-oriented climate was primarily and positively related to autonomy, competence, and relatedness need satisfaction. When students reported that their teacher put stronger emphasis on effort and personal improvement in the teaching units, they were also more likely to report that they were allowed to select the degree of difficulty of their activities, and to work at their own pace (autonomy satisfaction), that they were provided with opportunities to perform according to their capacities and individual skills (competence satisfaction), and to cooperate and share their own progress with their classmates (relatedness satisfaction).

Moreover, in line with SDT, and previous studies on PE (Haerens et al., 2015; Holt et al., 2018; Säfvenbom et al., 2015; Sánchez-Oliva et al., 2014), our results revealed that BPN satisfaction was positively related to autonomous motivation, which in turn was positively related to affective attitudes. The results suggest that selfdetermined reasons to participate in PE lessons play a significant role in the interest and usefulness of PE. BPN satisfaction was also negatively related to amotivation, which is consistent with SDT and most of the studies (e.g., Standage et al. 2005). Additionally, 
1 we found a significant cross-path between autonomous motivation and boredom, which

2 agrees with previous studies in the PE context (Sánchez-Oliva et al. 2014), and between

3 amotivation and affective attitude (Aelterman et al., 2012). However, we did not find a

4 significant cross-path between BPN satisfaction and external regulation. The absence of

5 a relationship between BPN satisfaction and external regulation is not in line with

6 previous studies on PE lessons (Haerens et al. 2015; Standage et al. 2005). A possible

7 explanation could be that, although the BPN were satisfied, attending PE lessons could

8 be perceived by students as a mandatory requirement.

9 The "dark" side of motivation.

10 An ego-oriented climate is characterized by teachers using statements such as

11 "You have to perform better than your classmates", "The best track train will obtain the

12 best grades”. In ego-oriented climates, teachers will more likely rely on public feedback

13 and evaluation in front of the whole class, which will probably produce social

14 comparison (e.g., "Sarah, you were the last to arrive"), and will generate excessive

15 competition among students. They also tend to spend more time with students who

16 perform better. No studies to date have analyzed the relations between an ego-oriented

17 climate and need frustration. In the current study, we found that an ego-oriented climate

18 related primarily to amotivation and negative affective outcomes via need frustration,

19 hereby confirming Hypothesis 1b. When students reported that their teacher emphasized

20 normative comparison and doing better than others, and displayed conditional regard

21 (i.e., spending more time with students who perform better), students were more likely

22 to feel pressured or pushed to satisfy the teacher's expectations (autonomy frustration),

23 to feel incompetent and unsuccessful (competence frustration), and to experience

24 alienation from others (relatedness frustration) (Holt et al. 2018). Moreover, congruent

25 with prior studies in PE lessons, BPN frustration was positively related to amotivation 
1 (Haerens et al. 2015), which in turn was positively related to boredom (Sánchez-Oliva

2 et al. 2014). Students, who feel pressured to participate, incompetent in their abilities

3 and that their classmates are cold and distant, are likely to consider the teaching units as

4 a waste of time and boring.

In addition, consistent with the findings reported by Martinent, Guillet-Descas,

6 and Moiret (2015) in a sample of adolescent athletes, we found a null-relation between

$7 \quad$ BPN frustration and external regulation. This could be explained because experiencing

8 need frustration in a specific teaching unit does not immediately seem to translate into

9 pressured functioning (i.e., participating in the unit because it is mandatory, to get good

10 grades, to please the teacher or to avoid punishment, etc.). Further, more recurrent

11 experiences of need frustration are needed to elicit controlled functioning. More studies

12 are required to discern the role of different motivational subtypes, distinguished in SDT,

13 with BPN frustration in PE lessons. Finally, contrary to previous studies in the PE

14 setting (Sánchez-Oliva et al. 2014), the students' external regulation was negatively

15 related to boredom. One possible reason for this unexpected result is that the present

16 work, unlike other studies, has focused on the students' perception of motivational

17 climate and motivational experiences during a specific teaching unit (e.g., rope

18 skipping, acrosport, soccer, athletics, badminton). To illustrate this, students sometimes

19 engage in PE teaching units because it is something that they have to do, but when they

20 participate in the unit activities or tasks, they may feel less bored if the activities were

21 attractive.

22 Cross-paths in the "bright" and "dark" side of motivation.

We also explored the possibility of specific cross-paths between motivational

24 climates, students' BPN, and motivational outcomes. The hypothesized negative cross-

25 paths from task-oriented climate to autonomy, competence, and relatedness frustration 
1 were fully supported (Hypothesis 2a). This suggests that a task-oriented climate could

2 also act as a buffer against need frustration. As pointed out by Vansteenkiste and Ryan

3 (2013), need-supportive environments can also play a buffering role against

4 malfunctioning and ill-being. Nevertheless, the symmetrical relationship between task-

5 oriented climate and need satisfaction was stronger than the cross-path relationship

6 between task-oriented climate and need frustration (see also Haerens et al. 2015; Liu et

7 al. 2017). The cross-path relationship between an ego-oriented climate and need

8 satisfaction was not significant (Hypothesis 2b), suggesting that to foster need

9 satisfaction, more is needed than just refraining from installing an ego-oriented climate.

10 Teachers probably need to actively install a task-oriented climate.

11 Finally, when inspecting the relations with each of the needs separately, students'

12 perceptions of ego-oriented climate were only negatively related to relatedness

13 satisfaction while no significant relationships were found with autonomy and

14 competence satisfaction (see also Álvarez et al., 2012; Reinboth and Duda, 2006). In an

15 ego-oriented climate, success in the task is defined in terms of outperforming others.

16 This likely generates an atmosphere of individual comparison among students where

17 students are no longer cooperating to achieve the objectives, which would not only lead

18 to alienation among students (i.e., relatedness frustration), but would also prevent them

19 from building positive relationships among each other (i.e., relatedness satisfaction).

20 Regarding the lack of relationship with the need for competence, it is possible that

21 students do not feel less or more effective when there is an ego-oriented climate. Yet,

22 the functional significance of an ego-oriented climate may also differ depending on

23 students' personal traits such as their actual motor competence. Because students with

24 high motor competence get more positive attention from the teacher, they may feel more

25 competent in an ego-oriented climate, competence satisfaction may be hampered 
1 (Álvarez et al. 2012; Reinboth and Duda 2006), particularly for less skilled students.

2 Similarly, some students, particularly those who are favored by the teacher, may

3 experience more autonomy to make decisions and provide input, while others might not

4 have their need for autonomy fulfilled. However, at this stage, these assumptions are

5 highly speculative and warrant further investigation. Nevertheless, it is not

6 recommended to install an ego-oriented climate since most studies, so far (Braithwaite

7 et al. 2011), display its detrimental effects, as was also the case in the current study, as

8 positive relations with need frustration and negative outcomes were found.

$9 \quad$ Practical implications

10 This study points to the importance of creating a task-oriented climate because

11 of the positive effects it can have on students' motivational experiences and learning in

12 PE lessons. According to Ames (1992), a task-oriented climate can be created when

13 teachers rely on the TARGET dimensions (i.e., task, authority, recognition, grouping,

14 evaluation, and time). Each of these dimensions can indeed be linked to BPN

15 satisfaction (for a further review, see Morgan 2017). For example, when teachers offer

16 students task choices (e.g., the task difficulty level) or provide opportunities for

17 decision-making and leadership during the teaching units (i.e., authority), students will

18 more likely experience autonomy satisfaction. Moreover, when evaluation includes

19 positive and individual feedback about the progress students have made, or a private

20 evaluation focused on progress and self-referenced standards (i.e., evaluation), and

21 students receive sufficient time to learn (i.e., time), their feelings of competence will be

22 nurtured. Finally, teachers can create heterogeneous and cooperative groups where each

23 student performs a defined role to help the other students to improve their abilities (i.e.,

24 grouping). This would then foster relatedness satisfaction. As such, the development of

25 interventions grounded in the six TARGET areas proposed by Ames (1992) could help 
1 to create a task-oriented climate in different teaching units (see Abós et al. 2017; Sevil

2 et al. 2016, for a further review). Hastie, Johnson, and Rudisill (2018) also provided

3 practical implications or elements (e.g., novelty, variety, progress point outs,

4 complexity, etc.) on how to design activities in task-oriented climates in PE classes. For

5 example, according to these authors, task complexity needs to be organized

6 hierarchically by the teacher and his/her students (e.g., Sarah could modify the

7 complexity of a task because her skills were below those demanded by the task).

This study also expands findings of previous studies, showing that task-oriented

9 climate could act as a buffer against BPN frustration. Moreover, it seems crucial that

10 teachers not only implement strategies to support a task-oriented climate, but also avoid

11 creating an ego-oriented climate in PE lessons (for a further review see Rudisill 2016).

12 For example, a competition where students are challenged to compete against each other

13 is inherent to a PE context, yet, there is no need for teachers to overemphasize who has

14 won the game or pay more attention to students who perform best.

15 Limitations and directions for future research

16 The present study has several limitations, and future research directions follow

17 from this study. First, this study involved 22 classes taught by 9 different teachers.

18 Some of the teachers taught only 1 class, while others taught 5 classes. Future studies

19 should increase the number of teachers involved and unify the number of classes taught

20 by the same teacher to increase external validity. Second, these findings have to be

21 interpreted with caution because all the data were based on students' self-reported

22 questionnaires. Future research using a mixed-methods approach to triangulate results

23 (i.e., teachers' and students' perceptions of task and ego-oriented climate, and

24 motivational climate observation) is needed. The inclusion of systematic observation

25 instruments to assess teachers' behavior in terms of motivational climate would be 
1 helpful to gain more insight into the frequency and intensity at which task- and ego-

2 oriented climates occur (Milton et al. 2018). Third, as the study was cross-sectional, no

3 causality can be inferred from the findings. Experimental or longitudinal designs are

4 needed in order to test the directional and bidirectional nature of the proposed

5 relationships. Fourth, the questionnaire to assess situational motivation did not include

6 introjected regulation. As in previous studies (Haerens et al. 2015), it might be

7 interesting to integrate this type of regulation to permit the calculation of a composite

8 score for controlled motivation.

9 Finally, previous studies suggested that perceived task- and ego-oriented

10 climates are two independent dimensions (Soini et al. 2014). It would be interesting to

11 establish motivational climates profiles to analyze how retained profiles (e.g., low or

12 high scores in both climates, high task-oriented climate and low ego-oriented climate, or

13 vice-versa) differ in BPN satisfaction and frustration, and students' motivation. In

14 addition, there might be other social factors that influence students' “dark” and "bright”

15 experiences in PE. For example, the inclusion of students' perception of peer

16 motivational climate in PE lessons might help to distinguish the role of teachers and

17 peers, separately.

\section{Conclusions}

19 The present study showed that experiences of need satisfaction and frustration

20 may explain why a task-oriented climate elicits positive outcomes, and that experiences

21 of need frustration account for the negative motivational impact of an ego-oriented

22 climate. The study highlights the importance of creating a task-oriented climate in PE

23 lessons due to its dual role in students' BPN satisfaction and frustration.

24

25 References 
1 Abós, Á., J. Sevil, J. A. Julián, A. Abarca-Sos, and L. García-González. 2017. “Improving Students' Predisposition towards Physical Education by Optimizing their Motivational Processes in an Acrosport Unit.” European Physical Education Review 23: 444-460. doi:10.1177/1356336X16654390

Aelterman, N., M. Vansteenkiste, H. Van Keer, L. Van den Berghe, J. De Meyer, and L. Haerens. 2012. “Students' Objectively Measured Physical Activity Levels and Engagement as a Function of Between-Class and Between-Student Differences in Motivation toward Physical Education.” Journal of Sport \& Exercise Psychology 34: 457-480. doi:10.1123/jsep.34.4.457

Álvarez, M. S., I. Balaguer, I. Castillo, and J. L. Duda. 2012. “The Coach-Created Motivational Climate, Young Athletes' Well-Being, and Intentions to Continue Participation.” Journal of Clinical Sport Psychology 6: 166-179. doi:10.1123/jcsp.6.2.166

Ames, C. 1992. “Achievement Goals and the Classroom Motivational Climate.” In Student Perceptions in the Classroom, edited by J. Meece and D. Schunck, 327348. Hillsdale, NJ: Erlbaum.

Baena-Extremera, A., A. Granero-Gallegos, C. Bracho-Amador, and F. J. Pérez-Quero. 2012. "Spanish Version of ohe Sport Satisfaction Instrument (SSI) Adapted to Physical Education.” Journal of Psychodidactics 17: 377-395. doi:10.1387/ Rev.Psicodidact.4496

Bartholomew, K., N. Ntoumanis, R. M. Ryan, and C. Thøgersen-Ntoumani. 2011. "Psychological Need Thwarting in the Sport Context: Assessing the Darker Side of Athletic Experience.” Journal of Sport and Exercise Psychology 33: 75102. doi:10.1123/jsep.33.1.75 
1 Biddle, S. J., F. Cury, M. Goudas, P. H. Sarrazin, J. P. Famose, and M. Durand. 1995. "Development of Scales to Measure Perceived Physical Education Class Climate: A Cross-National Project.” British Journal of Educational Psychology 65: 341-358. doi:10.1111/j.2044-8279.1995.tb01154.x

Braithwaite, R., C. M. Spray, and V. E. Warburton. 2011. "Motivational Climate Interventions in Physical Education: A Meta-Analysis.” Psychology of Sport and Exercise 12: 628-638. doi:10.1016/j.psychsport.2011.06.005

Cera, E., B. J. A. Almagro, C. Conde, and P. Sáenz-López. 2015. “Inteligencia Emocional y Motivación en Educación Física en Secundaria.”[Emotional Intelligence and Motivation in Secondary Physical Education.] Retos: Nuevas Tendencias en Educación Física, Deporte y Recreación 27: 8-13.

Chen, W. 2014. "Psychological Needs Satisfaction, Motivational Regulations and Physical Activity Intention among Elementary School Students.” Educational Psychology 34: 495-511. doi:10.1080/01443410.2013.822959

Deci, E. L., and R. M. Ryan. 2000. "The "What”' and "Why”' of Goal Pursuits: Human Needs and the Self-Determination of Behavior.” Psychological Inquiry 11: 227268. doi:10.1207/S15327965PLI1104_01

Di Battista, R., C. Robazza, M. C. Ruiz, M. Bertollo, F. Vitali, and L. Bortoli. 2018. “Student Intention to Engage in Leisure-Time Physical Activity: The Interplay of Task-Involving Climate, Competence Need Satisfaction and Psychobiosocial States in Physical Education.” European Physical Education Review. Advance online publication. doi:10.1177/1356336X18770665

Duda, J. L., and J. G. Nicholls. 1992. "Dimensions of Achievement Motivation in Schoolwork and Sport.” Journal of Educational Psychology 84: 290-299. doi:10.1037/0022-0663.84.3.290 
1 Erturan-İlker, G., and G. Demirhan. 2013. "The Effects of Different Motivational Climates on Students' Achievement Goals, Motivational Strategies and Attitudes toward Physical Education.” Educational Psychology 33: 59-74. doi:10.1080/01443410.2012.707613

Guay, F., R. J. Vallerand, and C. Blanchard. 2000. "On the Assessment of State Intrinsic and Extrinsic Motivation: The Situational Motivation Scale (SIMS).” Motivation and Emotion 24: 175-213. doi:10.1023/A:1005614228250

Gutiérrez, M., L. M. Ruiz, and E. López. 2011. “Clima Motivacional en Educación Física: Concordancia entre las Percepciones de los Alumnos y las de sus Profesores.” [Motivational Climate in Physical Education: Correlates between Students' and their Teachers' Perceptions.] Revista de Psicología del Deporte 20: 321-335.

Haerens, L., N. Aelterman, M. Vansteenkiste, B. Soenens, and S. Van Petegem. 2015. “Do Perceived Autonomy-Supportive and Controlling Teaching Relate to Physical Education Students' Motivational Experiences Through Unique Pathways? Distinguishing Between the Bright and Dark Side of Motivation.” Psychology of Sport and Exercise 16: 26-36. doi:10.1016/j.psychsport. 2014.08.013

Haerens, L., D. Kirk, G. Cardon, I. D. Bourdeaudhuij, and M. Vansteenkiste. 2010. "Motivational Profiles for Secondary School Physical Education and its Relationship to the Adoption of a Physically Active Lifestyle among University Students.” European Physical Education Review 16(2): 117-139. doi:10.1177/1356336X10381304

Haerens, L., C. Krijgsman, A. Mouratidis, L. Borghouts, G. Cardon, and N. Aelterman. 2018. "How does Knowledge about the Criteria for an Upcoming Test relate to 
Adolescents' Situational Motivation in Physical Education? A SelfDetermination Theory Approach.”European Physical Education Review. Advance online publication. doi:10.1177/1356336X18783983

Hastie, P. A., J. L. Johnson, and M. E. Rudisill. 2018. “An Analysis of the attraction and Holding Power of Motor Skill Stations used in a Mastery Motivational Physical Education Climate for Preschool Children.” Physical Education and Sport Pedagogy 1: 37-53. doi:10.1080/17408989.2017.1341476

Hilland, T. A., G. Stratton, D. Vinson, and S. Fairclough. 2009. “The Physical Education Predisposition Scale: Preliminary Development and Validation.” Journal of Sports Sciences 27: 1555-1563. doi:10.1080/02640410903147513

Holt, A. D., S. Smedegaard, C. S. Pawlowski, T. Skovgaard, and I. B. Christiansen. 2018. Pupils' Experiences of Autonomy, Competence and Relatedness in 'Move for Well-Being in Schools' A Physical Activity Intervention. European Physical Education Review. Advance online publication. doi:10.1177/1356336X18758353

Hu, L., and P. M. Bentler. 1999. “Cutoff Criteria for Fit Indexes in Covariance Structure Analysis: Conventional Criteria Versus New Alternatives.” Structural Equation Modeling 6: 1-55. doi:10.1080/10705519909540118

Jaakkola, T., S. Yli-Piipari, V. Barkoukis, and J. Liukkonen. 2017. "Relationships among Perceived Motivational Climate, Motivational Regulations, Enjoyment, and PA Participation among Finnish Physical Education Students.” International Journal of Sport and Exercise Psychology 15: 1-18. doi:10.1080/1612197X.2015.1100209

Liu, J., K. Bartholomew, and P. K. Chung. 2017. "Perceptions of Teachers' Interpersonal Styles and Well-Being and Ill-Being in Secondary School Physical 
Education Students: The Role of Need Satisfaction and Need Frustration.” School Mental Health 9: 360-371. doi:10.1007/s12310-017-9223-6

Marôco, J. 2014. “Análise de Equações Estructurais. Fundamentos Teóricos, Software \& Aplicações”. Pero Pinheiro: Report Number.

Martín-Albo, J., J. L. Nuñez, and J. G. Navarro. 2009. "Validation of the Spanish Version of the Situational Motivation Scale (EMSI) in the Educational Context.” The Spanish Journal of Psychology 12: 799-807.

Martinent, G., E. Guillet-Descas, and S. Moiret. 2015. “A Reciprocal Effects Model of the Temporal Ordering of Basic Psychological Needs and Motivation.” Journal of Sport and Exercise Psychology 37: 117-126. doi:10.1123/jsep.2014-0093

Milton, D., P. R. Appleton, A. Bryant, and J. L. Duda. 2018. "Initial Validation of the Teacher-Created Empowering and Disempowering Motivational Climate Questionnaire in Physical Education.” Journal of Teaching in Physical Education 37: 340-351. doi:10.1123/jtpe.2018-0119

Moreno, J. A., D. González-Cutre, M. Chillón, and N. Parra. 2008. “Adaptación a la Educación Física de la Escala de las Necesidades Psicológicas Básicas en el Ejercicio (BPNES).” [Adaptation of the Basic sychological Needs in Exercise Scale to Physical Education.] Revista Mexicana de Psicología 25: 295-303.

Morgan, K. 2017. “Reconceptualizing Motivational Climate in Physical Education and Sport Coaching: An Interdisciplinary Perspective.” Quest 69: 95-112. doi:10.1080/00336297.2016.1152984

Muñiz, J., P. Elosua, and R. K. Hambleton. 2013. “International Test Commission Guidelines for Test Translation and Adaptation.” Psicothema 25: 151-157. doi:10.7334/psicothema2013.24 
1 Nicholls, J. G. 1989. “The Competitive Ethos and Democratic Education.” Cambridge,

3 Ommundsen, Y., and S. E. Kvalø. 2007. “Autonomy-Mastery, Supportive or 4 MA: Harvard University Press. Performance Focused? Different Teacher Behaviors and Pupils' Outcomes in Physical Education.” Scandinavian Journal of Educational Research 51: 385413. doi:10.1080/00313830701485551

Reinboth, M., and J. L. Duda. 2006. "Perceived Motivational Climate, Need Satisfaction and Indices of Well-Being in Team Sports: A Longitudinal Perspective.” Psychology of Sport and Exercise 7: 269-286. doi:10.1016/j.psychsport.2005.06.002

Rudisill, M. E. 2016. "Mastery Motivational Climates: Motivating Children to Move and Learn in Physical Education Contexts.” Kinesiology Review 3: 157-169. doi:10.1123/kr.2016-0009

Säfvenbom, R., T. Haugen, and M. Bulie. 2015. “Attitudes Toward and Motivation for PE. Who Collects the Benefits of the Subject?” Physical Education and Sport Pedagogy 20: 629-644. doi:10.1080/17408989.2014.892063.

Sánchez-Oliva, D., P. A. Sánchez-Miguel, F. M. Leo, F. E. Kinnafick, and T. GarcíaCalvo. 2014. "Physical Education Lessons and Physical Activity Intentions Within Spanish Secondary Schools: A Self-Determination Perspective.” Journal of Teaching in Physical Education 33: 232-249. doi:10.1123/jtpe.2013-0043

Sevil, J., Á. Abós, A. Aibar, J. A. Julián, and L. García-González. 2016. “Gender and Corporal Expression Activity in Physical Education: Effect of an Intervention on Students' Motivational Processes.” European Physical Education Review 22: 372-389. doi:1010.1177/1356336X15613463 
1 Sicilia, A., R. Ferriz, and P. Saenz-Álvarez. 2013. "Validación Española de la Escala de frustración de las Necesidades Psicológicas (EFNP) en el Ejercicio Físico.” [Spanish Validation of the Psychological Needs Thwarting Scale in Exercise.] Psychology, Society \& Education 5: 1-19.

Smith, N., D. Tessier, Y. Tzioumakis, E. Quested, P. Appleton, P. Sarrazin, A. Papaioannou, and J. L. Duda. 2015. "Development and Validation of the Multidimensional Motivational Climate Observation System.” Journal of Sport and Exercise Psychology 37: 4-22. doi:10.1123/jsep.2014-0059

Soini, M., J. Liukkonen, A. Watt, S. Yli-Piipari, and T. Jaakkola. 2014. "Factorial Validity and Internal Consistency of the Motivational Climate in Physical Education Scale.” Journal of Sports Science \& Medicine 13: 137-144.

Standage, M., J. L. Duda, and N. Ntoumanis. 2005. “A Test of Self-Determination Theory in School Physical Education.” British Journal of Educational Psychology 75: 411-433. doi:10.1348/000709904X22359

Sun, H., W. Li, and B. Shen. 2017. "Learning in Physical Education: A SelfDetermination Theory Perspective.” Journal of Teaching in Physical Education 36: 277-291. doi:10.1123/jtpe.2017-0067

Vansteenkiste, M., and R. M. Ryan. 2013. “On Psychological Growth and Vulnerability: Basic Psychological Need Satisfaction and Need Frustration as a Unifying Principle.” Journal of Psychotherapy Integration 23: 263-280. doi:10.1037/a0032359.

Vlachopoulos, S. P., and S. Michailidou. 2006. "Development and Initial Validation of a Measure of Autonomy, Competence, and Relatedness in Exercise: The Basic Psychological Needs in Exercise Scale.” Measurement in Physical Education and Exercise Science 10: 179-201. doi:10.1207/s15327841mpee1003_4. 


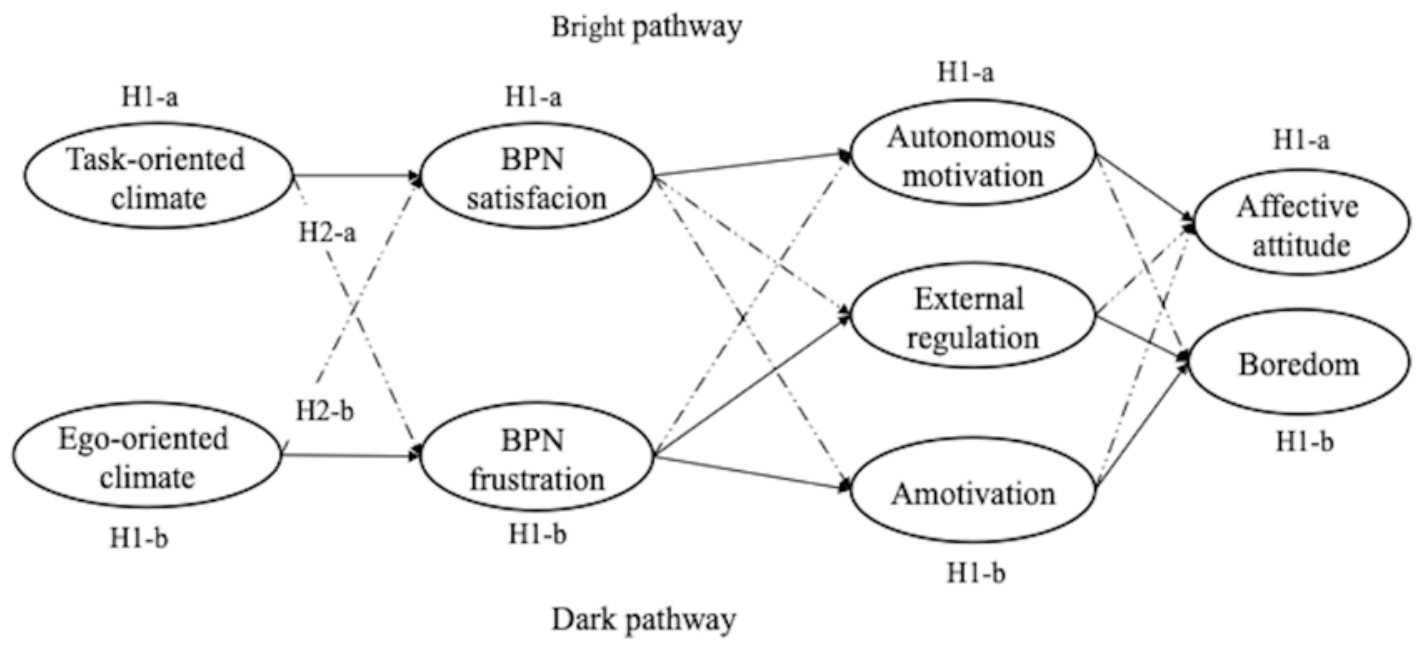

Figure 1. Hypothesized model of task- and ego-oriented climates, BPN satisfaction and frustration, motivational regulations, and affective outcomes. Continuous arrows showed positive relationships. Discontinuous arrows showed negative relationships.

Note: H1-a = Hypothesis 1a; H1-b = Hypothesis 1b; H2-a = Hypothesis 2a; H2-b = Hypothesis 2b. 


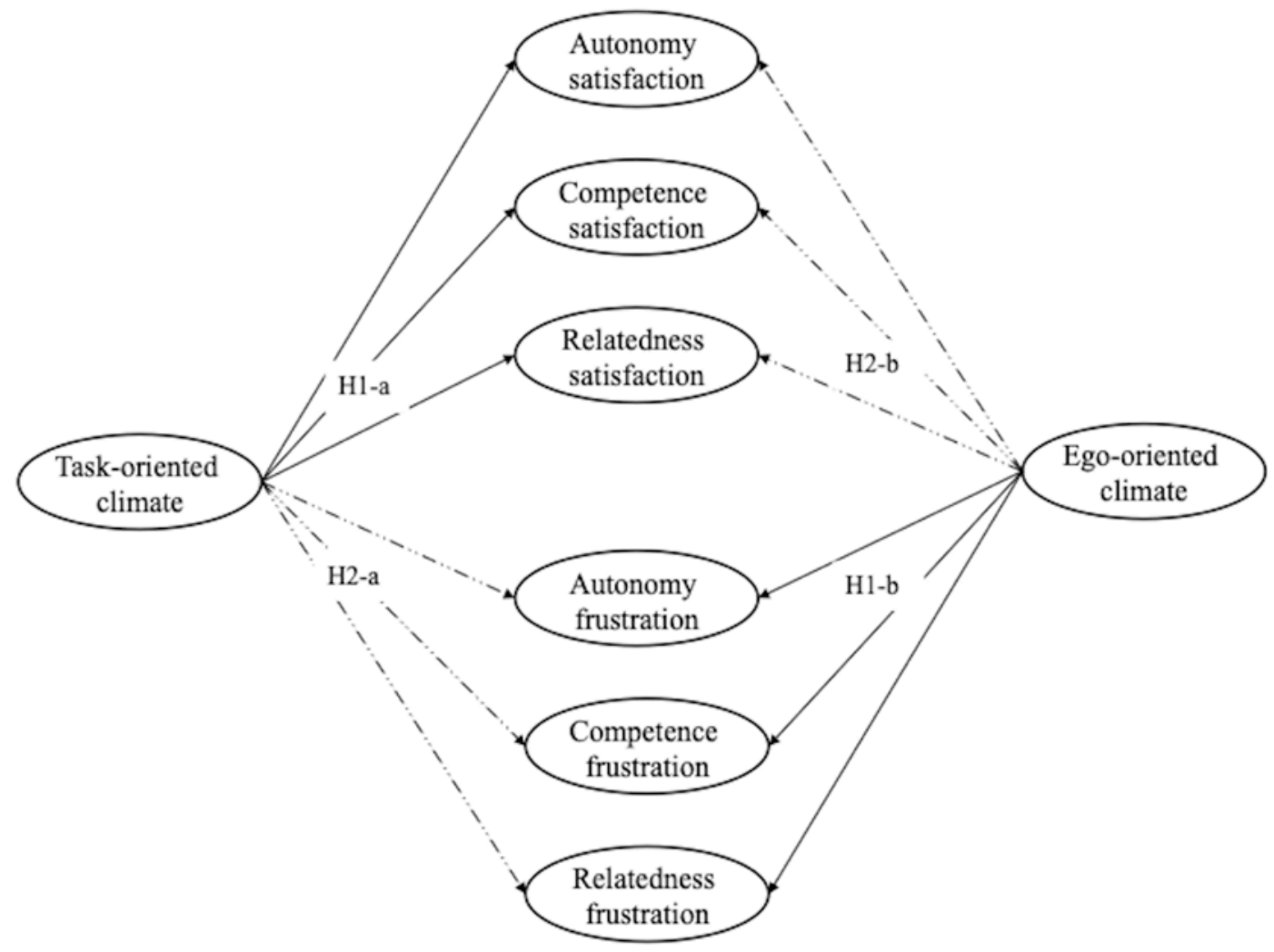

Figure 2. Hypothesized model of task- and ego-oriented climates and autonomy, competence, and relatedness satisfaction and frustration. Continuous arrows showed positive relationships. Discontinuous arrows showed negative relationships.

Note: H1-a = Hypothesis 1a; H1-b = Hypothesis 1b; H2-a = Hypothesis 2a; H2-b = Hypothesis 2b. 


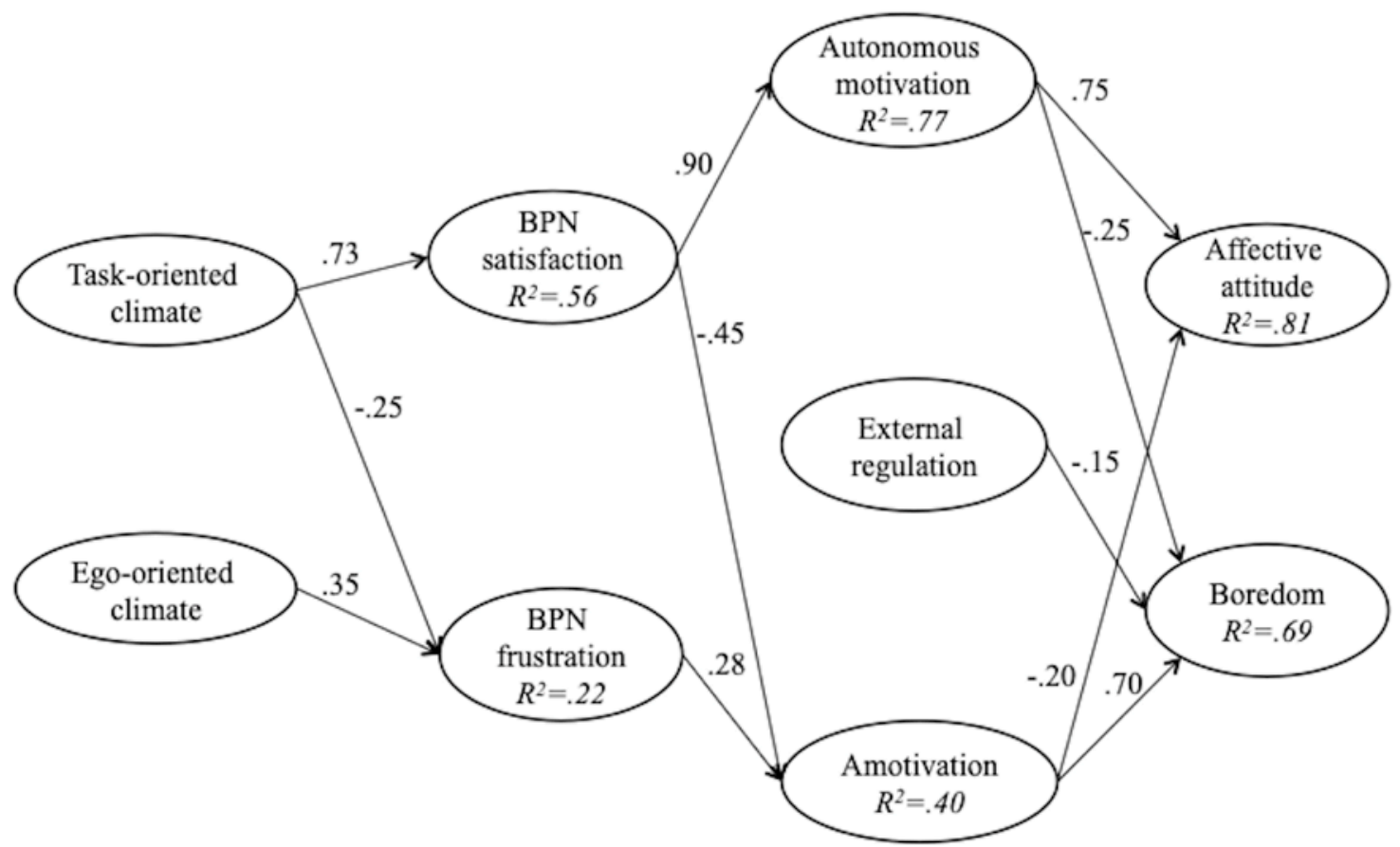

Figure 3. Structural equation model of task- and ego-oriented climates, BPN satisfaction and frustration, motivational regulations, and affective outcomes.

Note: Only significant paths are shown. All coefficients are significant $(p<.05)$. Standardized coefficients are reported 


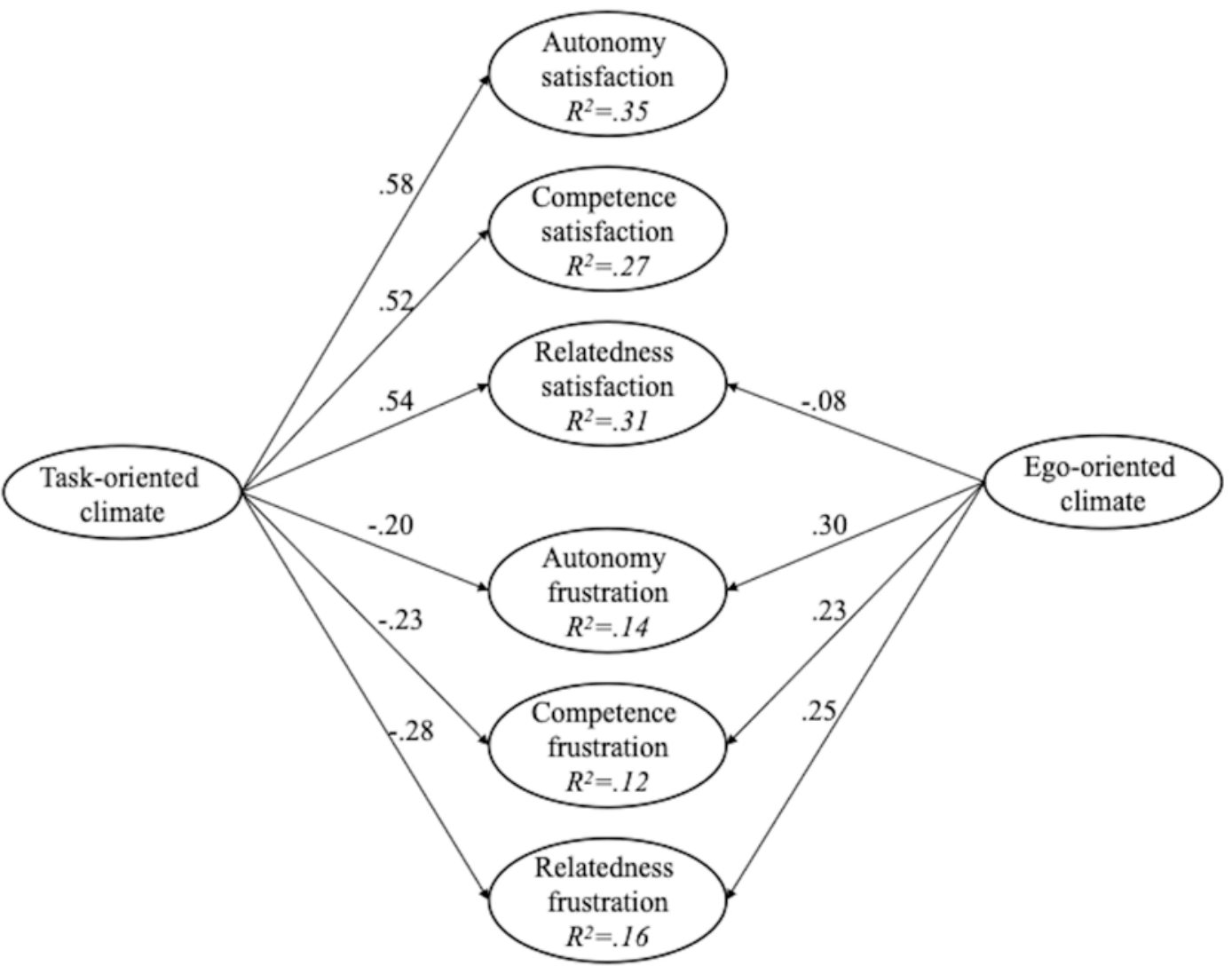

Figure 4. Structural equation model of task and ego-oriented climates and autonomy, competence, and relatedness satisfaction and frustration.

Note: Only significant paths are shown. All coefficients are significant $(p<.05)$. Standardized coefficients are reported. 